\title{
FRAGMENTO III: ACERCA DE LOS CONCEPTOS Y LAS DEFINICIONES
}

\author{
J. H. LAMBERT \\ Traducción, introducción y notas de Luciana Martínez ${ }^{1}$ \\ Universidad de Buenos Aires \\ Consejo Nacional de Investigaciones Cientificas y Técnicas \\ bttp://dx.doi.org/10.15304/ag.39.1.5907
}

\section{Introducción}

El artículo cuya versión en español presentamos aquí fue incluido en el volumen: Lambert, J. H. (1782) Logische und philosophische Abbhandlungen, Berlin, pp. 193-214. Es esta versión del texto la que ha sido empleada para la traducción. Por consiguiente, se indica en el texto el número de página correspondiente a esa edición en cada caso ${ }^{2}$.

El libro contiene el texto "Seis ensayos sobre una Zeichenkunst en la Lógica", y varios fragmentos sobre Lógica. El texto cuya traducción se encuentra a continuación es el tercero de estos fragmentos. El primero de

\section{Recibido: 20/03/2019. Aceptado: 11/06/2019.}

1 The project leading to this contribution has received funding from the European Union's Horizon 2020 research and innovation programme under the Marie SkłodowskaCurie grant agreement No 777786.

${ }^{2}$ El texto, además, ha sido reeditado y publicado en el siguiente volumen: J. H. Lambert, Philosophische Schriften. 10 Bde. in 13 Bdn. Begonnen von Hans Werner Arndt. Fortgeführt von Lothar Kreimendahl. Band 6: Logische und philosophische Abhandlungen. Teil I. Berlin 1782. Georg Olms, Hildesheim, 1967. 
ellos, cuya traducción al español se encuentra en prensa, trata sobre el concepto de Lógica en general. El segundo trata sobre la facultad del entendimiento. El tercero introduce la doctrina de los conceptos y sus definiciones.

Esta traducción ha intentado ser literal y mantener hasta los signos de puntuación del texto original. Se han alterado, empero, algunos signos, con el fin de alcanzar un texto legible en español. La elección de algunas palabras de esta lengua debe ser comentada. Hemos decidido traducir los términos "Sache" y "Ding" como "cosa"; "Erklärung", como "definición"; "Bestimmung" como "determinación".

\section{Acerca de los conceptos y las definiciones ${ }^{3}$}

\section{[193]}

1. Una nota es la representación de algo, por medio de la cual distinguimos una cosa que contiene ese algo de las demás [cosas]. Es compuesta, si admite descomponerse en [otras] más simples; simple, si no admite descomponerse; interna, si está constantemente en la cosa; externa, si no está constantemente en la cosa.

Un concepto es la representación de la cosa en el pensamiento; reúne en sí todas las notas de ella, seamos conscientes de ello o no. Una cara de la cosa contiene aquellas notas que van juntas, si nos las representamos con una determinada intención. La cosa tiene tantas caras como intenciones según las cuales nos la podamos representar. Es claro que hay notas que pueden darse en más de una cara.

[194]

Nuestro conocimiento tiene diferentes grados de oscuridad, hasta la mayor distinción y detalle. Un concepto es oscuro si no podemos representarnos la cosa; es claro, si, sin reconocer sus notas, podemos [representarnos la cosa]. Si pensamos claramente las notas, el concepto es distinto. Si tenemos conceptos distintos de las notas, es detallado.

Todo esto tiene diferentes grados. Un grado, por ejemplo, separa los conceptos claros, distintos o detallados, de los que no lo son. Un concepto claro es suficientemente claro, si podemos reconocer la cosa por medio de los sentidos en todas las circunstancias; es suficientemente distinto, si podemos reconocer de esa manera sus notas, etc. Trazamos esta diferencia, no como si la distinción no pudiera ser perfeccionada; siempre podemos encontrar

${ }^{3}$ El mismo título y un contenido semejante tiene el primer capítulo de la "Dianología", que es el primer libro del Neues Organon, de 1764. 
nuevas notas y volverlas claras, provisional o suficientemente; sino porque la distinción suficiente es la extensión [Umfang] del conocimiento erudito. Podemos denominar la cara aclarada a aquellas notas de una cosa que pertenecen al concepto distinto de ella.

Dos conceptos son iguales si tienen las mismas notas. Nos presentan la misma cosa, sólo que de diferentes maneras. Siempre podemos poner uno en el lugar del otro. Son parecidos (coordinados), si sólo algunas de sus notas son las mismas; emparentados (subordinados), si uno es una nota del otro [195]. Éste tiene todas las notas de aquél, y algunas más. Los parecidos tienen, cada uno, algo propio. Así que los conceptos parecidos y los emparentados siempre son diferentes en algo entre sí.

2. Esta es la clasificación general de los conceptos. La clasificación general de las notas las divide en: 1) esenciales, 2) propias, 3) comunes. En cada concepto hay, pues, notas esenciales, que son las que les corresponden individualmente y que los diferencian de otros; propias, que en la comparación con otro, a ellos solos les corresponden; comunes, si les corresponden a la vez, de modo que puedan ser comparados. A partir de estas definiciones se siguen las siguientes proposiciones:

a. Los conceptos iguales tienen notas esenciales iguales. Los conceptos iguales representan cosas iguales, pueden reemplazarse entre sí, tienen notas iguales en general.

b. Las notas propias y las comunes constituyen el concepto completo.

c. Dados dos conceptos parecidos, si sacamos las notas propias de cada uno, de modo que sólo queden las comunes, se siguen conceptos iguales.

d. Si se sacan las notas propias de un concepto A, y en su lugar [196] se agregan las propias de B, entonces resulta el concepto B.

e. Si se sacan las notas propias de uno de los conceptos emparentados, queda el otro.

3. A partir de esta teoría podemos derivar la descomposición de los conceptos y mostrar cómo debe verse el edificio completo mismo. Las notas esenciales son las que pertenecen a un concepto de manera completamente individual; si las sacamos de un concepto, sólo quedan las que tiene en común con otros conceptos. Tomadas todas juntas, éstas forman otro concepto, es decir, el género [Gattung] de aquél; sus notas esenciales reunidas, son la diferencia de especie ${ }^{4}$. De aquí se sigue que esa diferencia y el género

\footnotetext{
${ }^{4}$ En esta oración hemos alterado significativamente la puntuación escogida por Lambert.
} 
reunidos constituyen el concepto completo. Si se los expresa con palabras, se denominan una definición. Una definición verdadera y esencial consiste pues en el género y la diferencia de especie .

El género puede ser visto como un concepto, así que puede buscarse a su vez su género y la diferencia de especie , y así infinitamente. Una especie, vista desde una especie ínfima, se denomina género. Las especies ínfimas no tienen bajo sí más que individuos. El verdadero sistema de los conceptos parece ser semejante a un árbol genealógico, cuyas ramas parecen seguir al infinito.

[197] Es posible que no tengamos ni una definición de éstas [que sea] perfecta. Los géneros y las especies de los conceptos se elevan gradualmente, tan imperceptiblemente, que cada diferencia de especies consiste en notas simples, comienza infinitamente pequeña y se acumula por medio de aumentos infinitamente pequeños. Cuando clasificamos los conceptos en especies y géneros, consideramos juntos, por así decirlo, familias completas y generaciones y las diferenciamos en especies. Las agrupamos con el resto de las generaciones de la misma raíz, y llamamos a esto el género.

4. De los conceptos emparentados A y B, siempre uno tiene notas propias, por ejemplo A. Ellas faltan en el concepto B. Si se añaden esas notas al concepto B, se completa la falta y el concepto así compuesto es equivalente a A. A aquél lo llamamos la definición, a éste el definido. B el género; el suplemento, la diferencia de especie. Esta clase de definición es aún la más aceptable. Pero se ve también que según este método pueden hacerse infinitamente muchas definiciones de uno y el mismo concepto. El concepto A tiene infinitamente muchas notas, se puede abstraer ya una, ya la otra de ellas y combinarlas infinitamente. Cada combinación representa el concepto $\mathrm{B}$, y éste es el género del concepto $\mathrm{A}$. Las notas que todavía no se han combinado en un nuevo concepto, reunidas, [198] son la diferencia de especie; reúnanse ambas, y se tiene una definición. La cantidad de definiciones posibles es igual a la cantidad de combinaciones posibles, y ésta es infinita. No quiero desestimar con esto esas definiciones. No podemos hacer que sean mejores. Las diferentes definiciones de una cosa nos dan conceptos intercambiables [Wechselbegriffe], que son extraordinariamente útiles y nos hacen falta. Sólo debe demostrarse la corrección de tales definiciones, o, si ya está demostrada, deben derivarse de ellas las demás. Frecuentemente es posible derivar más de una definición que de las otras, es más fácil derivar lo que trabajosamente se obtuvo a partir de otras. La una puede ser teórica; la otra, práctica; una sirve para descubrir las causas; la otra, para otras investigaciones. Este tipo de definiciones proporciona menos notas reales. 
5. En cambio, cuando A y B son parecidos, no sólo hay que añadir en $\mathrm{B}$ las notas propias de A, sino que también hay que sacar las notas propias de B, para que uno pueda definir el uno con el otro. Estas definiciones son de escaso valor en las ciencias, pero en las cosas prácticas es bueno usarlas, se puede poner y sacar de B y de sus partes, y se tiene un concepto claro. El artista las emplea usualmente cuando quiere mostrar cómo se elabora una cosa a partir de sus materiales o cómo se convierte uno en el otro. Si, por ejemplo, quiero describir un higrómetro, [199] digo, en pocas palabras: "saque el platillo de una romana y cuelgue en su lugar una esponja", y la definición está lista ${ }^{5}$. El que tiene un concepto claro de la romana, puede representarse lo que se obtendrá.

6. Si A se define por medio del concepto pariente B y no A sino B tiene notas propias, entonces hay que sacarlas de $\mathrm{B}$, de modo que queda una definición privativa donde, si no, habría una mezclada.

7. Otra prueba de la imperfección de nuestras definiciones. Naturalmente el género debería contener todas las propiedades que la cosa definida tiene en común con las otras, y la diferencia de especie debe tener aquellas que sólo ella tiene. Sólo hay que ver si nuestras definiciones coinciden con estas reglas verdaderas. Se define al hombre como un animal racional. "Animal" es el género próximo; "racional", la diferencia de especie ; de modo que sólo el hombre debería ser racional. Sólo que todos los espíritus lo son. Pero incluso un espíritu podría pertenecer a otro género que el animal. Se restringen, de esta manera, las reglas mencionadas; la diferencia de especie no es una diferencia absoluta, sino que sólo diferencia una especie de las demás en un género; luego, la definición de arriba no dice que el hombre es lo único racional entre todas las cosas, sino entre todos los animales de la Tierra. Pero esa [200] restricción sola no ayuda. Pues cualesquiera sean las características del hombre que tome siempre del género o siempre de la diferencia de especies, no habrá ninguna cosa del género elegido que sea la única a la que corresponda completamente la diferencia de especie; por

${ }^{5}$ Considerado por algunos el inventor del higrómetro, Lambert publicó en 1769 el texto Essai d'bygrométrie, ou sur la mesure de l'bumidité, en el que menciona y describe algunos instrumentos de medición disponibles. Las primeras líneas de ese texto expresan la sorpresa de Lambert ante el hecho de que hayan sido desarrolladas diversas variantes de ese instrumento, ninguna de las cuales ha sido satisfactoriamente perfeccionada ( $\$ 1$ ). Además, introduce la noción del instrumento de medición a partir del concepto, conocido por todos, de la humedad, y enumera las utilidades de sus mediciones $(\$ 2)$. El libro es, por último, un detallado registro de mediciones realizadas por el mismo científico, ordenadas con precisión. 
consiguiente, tampoco [en el caso de] el hombre. Siempre faltarán aquellas notas que son absolutamente propias de él y que constituyen, empero, su diferencia de especie esencial en el verdadero sistema de las ideas.

De aquí, se aclara cuán poco conocido nos resulta este sistema de las ideas, por qué hay diferentes definiciones de algunas cosas, por qué colocamos algunas cosas bajo diferentes géneros y, no obstante, podemos ver tanto este género como el siguiente; se aclara, además, que nadie puede preferir sus definiciones a las otras por la razón de que su género y diferencia de especie estén mejor determinados, sin atender a que puede ser mejor según otros puntos de vista, como más fecundo, o más práctico. Por cierto, una definición puede estar más cerca de la perfección que las otras, sólo que esto es difícil de determinar en la mayoría de los casos y con mucha frecuencia es poco relevante porque con una perfección menor son más provechosas.

La forma usual de descomponer los conceptos en especies y géneros también es insuficiente. Pero como está regida por nuestra manera de representarnos las cosas podemos quedarnos con ella. Sirve para ordenar en cierta medida nuestros conceptos, sólo que no debemos considerar ese orden como el único, necesario [201], esencial; no debemos pensar que las definiciones deben basarse en ella, o que la cosa sólo tiene un único género, pues puede tener innumerables. En lo esencial, esa teoría no cambia en la doctrina de las inferencias. Se pueden admitir muchas definiciones y géneros, las inferencias no varían. Un género según nuestra determinación no es más que una propiedad que corresponde a otras cosas; el dictum de omni \& de nullo, el fundamento de las inferencias montado según estas denominaciones generales: "Lo que puede decirse de una propiedad, puede decirse de todas las cosas que tienen esa propiedad", permanece verdadero y correcto.

Denominamos "cara" a aquello que se ha considerado una propiedad o el género de una cosa; de modo que la diferencia de especie es siempre un apéndice de la cara.

Incluimos en una especie o un género todas las cosas que tienen entre sí un parecido llamativo perceptible por los sentidos, por ej. los diferentes géneros de animales, plantas, etc. En ellos se encuentra de manera directa el género mínimo casi con más fortuna que el superior; pues en ellos no es tan fácil encontrar la diferencia de especie . Cuanto más altos son los géneros que se buscan, tanto más libres en la elección nos dejan los sentidos, pues las diferencias de los géneros superiores no sobresalen tanto. En los conceptos abstractos, la elección es aún más libre. 


\section{[202]}

8. Las definiciones elaboradas según la teoría presentada se pueden separar en clases [Klassen]. En general, en esas definiciones buscamos primero el género. Para eso es aprovechable cualquier propiedad, y es más útil guiarse por la naturaleza de lo definido, de modo que hay substancia o accidente, según el concepto definido sea substancia o accidente. Así, el género será parecido al género esencial. Según esta teoría, podemos ver cada determinación de lo que el concepto tiene en común con otros como su género. Así obtenemos diferentes definiciones que lo son mucho más, cuando la diferencia de especie se vuelve más diferente. Sólo que estas últimas pueden ser diferentes todavía de otras maneras. Veamos, sea el género del concepto A determinado, a saber, B, uno puede tomar la diferencia de especie de diferentes maneras.

a. Para ello, uno puede tomar un concepto que contiene todas las otras notas de A, que no pertenecen a B. Estas definiciones son las más completas, pues ellas son absolutamente iguales al concepto definido, y en consecuencia contienen todas las notas que están bajo ese concepto.

b. Para la diferencia de especie se puede tomar un concepto tal que, entre todas las cosas que tienen la propiedad B, sólo [203] corresponde a la cosa A. Éste es el caso más usual de nuestras mejores definiciones, las hacemos con la intención de diferenciar la cosa A de todas las demás y logramos nuestra finalidad.

Cuando entre las cosas [Dingen] hay B o C, la cosa [Sache] A sola ocupa diferentes propiedades, entonces uno puede [tomar] todas juntas, o algunas, o una individual que se quiera para hacer la diferencia de especie. Las definiciones así creadas proporcionarán siempre una característica correcta de la cosa. Esas definiciones no dan la identidad absoluta de lo definido con la definición. Pero ésta siempre puede ser valorada como igual a lo definido, y en consecuencia puede ser usada como concepto intercambiable [Wechselbegriff]. A partir de allí, uno siempre puede hacer una proposición que al derecho y al revés sea en general afirmativa.

Si somos libres de hacer varias definiciones de la misma cosa, entonces está bien si las hacemos y las demostramos individualmente, o bien inferimos una a partir de las otras. En las ciencias, nada es de mayor utilidad que proposiciones que sean en general afirmativas al derecho y al revés. 
9. Todo eso sobre las diferentes clases de definiciones. Ahora, sobre el uso de tales clases.

Si se define una cosa para tornarla reconocible, entonces $\mathrm{B}$ y $\mathrm{C}$ deben corresponder permanentemente a la cosa $\mathrm{A}$, y tales son propiedades de las que uno tiene por lo menos conceptos claros. Además, B, como la primera característica, debe [204] corresponder a tan pocas cosas como sea posible, para que no haya que recorrer el mundo para encontrar C; pues C será más difícilmente determinada, cuanto más cosas haya que tengan la propiedad B. B y C no deberían ser propiedades esenciales, si no se tiene otro objetivo que poder diferenciar la cosa A de las demás por medio de los sentidos. En este caso, uno se conforma, como en la herboristería, con características externas. Pero si el concepto de la cosa ha de ser intelectual, entonces B y $\mathrm{C}$ deben ser propiedades esenciales, o [deben] hacer la cosa reconocible no sólo en la superficie, sino también en su corazón mismo.

Si se define una cosa para hacer demostraciones o fundamentar sus otras propiedades, entonces B y C deben ser conceptos que ofrecen un soporte fecundo, si de la definición se infieren principios [Grundsätze] y A se mantiene como sujeto. Para ser fecundo es útil, si dos conceptos de la cosa son igualmente amplios, en los casos en los que además se pueden presentar diferentes propiedades de $\mathrm{C}$ o de la diferencia de especie, colocar todas ellas bajo un concepto.

Si se define un concepto para la praxis, B y C deben ser tomados de aquel lado de la cosa de la cual uno pueda ver con mayor facilidad o inferir su génesis. Por ej. B muestra las partes; C, el tipo de composición o unión. B muestra la materia; C, la forma. B muestra la causa; C, el modo como actúa. B muestra el material del que surge A; C, la manera. B, el medio; C, el objetivo. Este tipo de definiciones puede denominarse práctico. También [205] las definiciones pueden tornarse prácticas cuando por ej. no expresan la causa, pero sí una propiedad que, como el efecto más distinguido de la causa, nos conduce a ella.

10. Uno no puede decir que un tipo de definición sea más fácil que el otro; en todos los tipos hay dificultades y facilidades, pero podemos, en términos generales, señalar lo siguiente.

a. Las definiciones del primer tipo (3.) parecen ser igualmente fáciles y difíciles en todas las ciencias, en la medida en que la cosa exprese más o menos sus notas.

b. Las definiciones del segundo tipo (4.) son más fáciles en aquellas ciencias que podemos demostrar a priori, es decir, en la Ciencia básica [Grund- 
wissenschaft], la Moral, la Lógica, la doctrina de las dimensiones [Grössenlehre], etc.

c. Las definiciones del tercer tipo, o prácticas, son más fáciles en aquellas cosas cuyas causas son nuestras acciones libres, en la medida en que nosotros las hacemos, y por lo tanto en todas las artes, en la medida en que son prácticas.

d. Las palabras que son abstracta de verbos y adjetivos admiten ser definidas según el segundo tipo (4.) con más facilidad que las palabras que [206] significan substancias efectivas [wirkliche] particularmente naturales; aquellas son perfección, magnitud, orden, etc.

e. Entre las definiciones prácticas, las más fáciles son aquellas que se obtienen de la finalidad [Absicht] la cosa. A este grupo pertenecen las definiciones de la mayoría de los instrumentos, máquinas, herramientas, etc. La finalidad está dada, y la cosa es el medio.

f. La más difícil de todas las ciencias, desde el punto de vista de las definiciones, es la Doctrina de la naturaleza y en ella, particularmente, el tipo del cuerpo y la materia, las causas naturales de muchos cambios, la estructura interna de las pequeñas partes, etc. Es además la única ciencia en la que sólo se puede concluir a posteriori.

Estas indicaciones sirven particularmente para mostrar el camino más fácil en el que debemos caer al meditar. El más fácil es al mismo tiempo el más natural. No podemos darnos más ni mejores sentidos, así que debemos conducirnos según la forma de ver las cosas que nos es natural. Quien quiera concluir o meditar en la Doctrina de la naturaleza como en las ciencias básicas, en las ciencias teóricas como en las prácticas, a priori como a posteriori, producirá, en lugar de nuevas verdades, o bien nada, o nada menos que sueños vacíos.

[207]

11. No sólo para las definiciones, sino para todo el conocimiento erudito son necesarios, como fue señalado, conceptos suficientemente claros: la primera cuestión de la Lógica es, pues, cómo obtener tales conceptos. En la mayoría de los casos, la respuesta no requiere más que procurar sentir con los sentidos las cosas de las cuales se deben obtener conceptos claros.

Aquí se encuentran dos cosas. La primera es la sensación de la cosa; la segunda, la lengua. La primera da al concepto claro la cosa que sentimos; la otra, el nombre. Éste no torna más clara la sensación, pero es indudablemente necesario; pues llevará la cosa a nuestros sentidos, siempre que lo repitamos, o escuchemos, o veamos. Rescata la claridad de la representa- 
ción de la cosa sentida, por así decirlo, de la extinción, del paulatino oscurecimiento. Debemos tener, pues, conceptos claros de ambos, de la palabra y de la cosa.

Para la obtención y el mantenimiento de los conceptos claros sirve la siguiente regla: "Procura sentir la cosa con tantos sentidos y en tantas circunstancias diferentes como sea posible, hasta que veas que volverías a reconocerla en todas las circunstancias; hazte conocido su nombre propio, piénsalo junto con la cosa y repite tanto la sensación como el nombre, tantas veces como sea posible”. Esta regla se puede utilizar con más facilidad para cosas que son substancias, que fácilmente caen en los sentidos, que tienen parecido con otras y se nos presentan con frecuencia.

[208] Ejercitamos esta regla desde jóvenes. Afortunados son los niños cuya madre, nodriza, o quien les enseñe a hablar, posea entendimiento y les enseñe tanto a conocer exactamente las cosas como a usar sus nombres exactos. Esto es en particular de gran importancia para la denominación de las acciones morales. Un niño ve, por ejemplo, una buena acción, y la nodriza, por ignorancia o afición le da a la persona o a la acción un mal nombre; ¿cómo podría alcanzar el niño un concepto correcto de la virtud y los vicios? Después uno tiene dificultad para hacer que el joven se comporte o mejore esos conceptos confusos. Las primeras impresiones tienen la fuerza de una certeza contundente, plantan prejuicios que arraigan tan profundamente que ya no se encuentra su origen. El que tiene conceptos erróneos de las palabras que usa no puede hacer nada mejor que comenzar nuevamente por la sensación, y será afortunado si encuentra un buen líder que le muestre las cosas significativas y le permita sentir.

Aumentamos la cantidad y la claridad de un concepto cuando fortalecemos los sentidos, es decir, por ejemplo, mejoramos la debilidad de los ojos con telescopios y con lentes de aumento.

Como para obtener conceptos claros hay que tener a mano alguien que nos pueda decir el nombre de las cosas sentidas o el significado de los nombres, y, sin embargo, esta ocasión nos falta, sería bueno si pudiéramos encontrar un método [209] o alguna señal por medio de la cual pueda descubrirse la cosa en ocasión de su visión, su nombre, o cuando escuchemos el nombre. En la Herboristería y en algunas otras partes de la Historia Natural ya se ha realizado este tipo de búsqueda, pero hasta ahora no se ha extendido este método.

Tornar claros las palabras y los conceptos a través de los otros requiere en las ciencias palabras artificiales y definiciones, también, en algunas ocasiones, figuras. En el trabajo del arte, cosas parecidas. Obtenemos las partes 
de aquello que pensamos con claridad, hasta llegar a la imagen de lo otro. Éste es el modo como artistas y artesanos hacen claros sus bocetos para los demás. Este método sólo nos trae las cosas compuestas cuyas partes podemos representarnos con claridad. Sin embargo, no debemos rechazarlo, no sólo porque por medio de él podemos enseñarles a los otros conceptos claros del todo, sino también porque la mayoría de los inventos del arte se basan en él. Un medio más general para obtener conceptos claros y distintos sería el arte general de los diagramas, si estuviera desarrollado. Pero en otras circunstancias.

Los conceptos distintos son aquellos, cuyas notas son claras. El arte de obtener conceptos distintos pertenece a la capacidad de hacer claras las notas. Esto no es nada menos que definir una palabra o concepto. No se busca más que hacer claras las notas $\mathrm{B}$ y $\mathrm{C}$ de $\mathrm{A}$.

[210] Los conceptos detallados son aquellos cuyas notas son distintas; el arte de hacer conceptos distintos pertenece por consiguiente a la capacidad de hacer distintas las notas. De esto hemos hablado en los apartados precedentes.

12. Se llega a los conceptos por medio de los nombres, ¿cómo [hacemos] cuando tenemos los conceptos y no tenemos nombres para ellos? En ese caso, o bien no hay un nombre que lo exprese y entonces hay que buscar uno. O bien hay un nombre, sólo que no se nos ocurre, pues nunca lo hemos pensado al mismo tiempo que al concepto; en este caso hay que revelar las notas de la cosa, hasta que lleguemos a la que alguna vez hayamos pensado junto con el nombre. En la doctrina de la naturaleza, si se necesitan frecuentemente líneas curvas, ellas se determinan, pero no por medio de las aplicaciones que nos permitirían reconocerlas. A partir de una ecuación, se deduce otra que define la naturaleza de la línea a través de las abscisas y las semi-ordenadas. Snell encontró la ley de la refracción, no reconoció la línea por la cual se representa. Descartes notó por primera vez que era el seno. Acontece con frecuencia que llegamos a la definición por reflexión, sin conocer la cosa definida o saber que tiene un nombre conocido. Este saber es siempre muy provechoso. Si conocemos muchas propiedades importantes de una cosa, sin conocerla por su nombre, aquellas nunca se encontrarán bajo él; hacemos entonces de una [211] cosa dos, y las propiedades que se siguen de la reunión de ambas se pierden para nosotros.

Para encontrar el nombre propio de cada concepto, primero, cuando se descubren al meditar aparentes nuevos conceptos, hay que ser desconfiado; segundo, hay que investigar si la cosa no es ya conocida. Esta investigación se puede reducir a las siguientes tareas. 
Sean dadas dos notas, B y C, del concepto A. La tarea se puede realizar de tres maneras, si B y C se consideran definiciones y se busca aquello que se va a definir. Se toma la nota $B$ y se examinan los conceptos a los que corresponde; entre ellos se buscan aquellos que son C; sus nombres serán los objetos que se busca definir. Después se procede de la misma manera con el concepto C. Finalmente se infieren las notas reunidas de los conceptos B y C que sean más estrechas, de modo que se encontrará entre ellas la que nos recuerde el nombre.

Si el concepto es realmente nuevo, entonces no tiene un nombre y hay que darle uno, si vale la pena. En la Matemática, donde se puede recurrir al Álgebra, es más fácil la resolución de esta tarea, pues hay definiciones algebraicas de las cosas y sus especies. Esta ventaja nos conducirá a procurar el arte de los diagramas en las otras ciencias, cuando él haya sido inventado. Pues por medio de él estaríamos en la situación no sólo de señalar [212] los conceptos, sino también de volver a reconocerlos a partir de los diagramas y sus conexiones.

13. Quien quiera construir un edificio doctrinal [Lehrgebäude] completo sobre una cosa, debe tener ante sí, desde el comienzo, todos los conceptos que le corresponden. Si esto no sucede, entonces le faltarán definiciones, le faltarán los principios construidos sobre ellas, le faltará la visión de conjunto, las conexiones. Ahora, para poder juzgar la completitud o incompletitud de los materiales reunidos en un sistema, pretendo estudiar con más detalle la naturaleza de los conceptos que pertenecen a un edificio doctrinal; veo de antemano que debe hacerse sintéticamente.

1. Le pertenece el concepto del objeto principal del tratado. 2. Los conceptos que están relacionados con él. 3. Los conceptos que se encuentran cuando se define el concepto principal, de tantos modos como sea posible. 4. Las partes físicas de la cosa, si tiene tales partes. 5. Las partes lógicas tanto de la cosa, como de sus especies, causas, propósitos, etc. 6. Los conceptos de sus cambios. 7. Aquello que podamos hacer con ellos. 8. Los efectos de la cosa en nosotros, en el entendimiento, la voluntad, los afectos y el cuerpo $[$ Leib]. 9. Las diferentes circunstancias en las que puede encontrarse la cosa. 10. Las diferentes determinaciones que uno puede hacer en ellas. 11. Los conceptos que surgen cuando se lleva la cosa por las fórmulas de las tareas y los principios lógicos y ontológicos, etc. 


\section{[213]}

14. Cuando se define una palabra, hay otras palabras que son definidas al mismo tiempo. 1. Todas las palabras verdaderamente equivalentes [gleichbedeutende]. 2. Todos los compuestos que se pueden formar con ellas. 3. Sus derivados. 4. Sus decomposita. Por ej., si hemos definido un verbum como "amar", entonces las definiciones de las siguientes palabras no serán difíciles: amado, el amor, amoroso [lieblich], amable [liebenswürdig], encanto [Lieblichkeit], enamorar, etc. 5. El oppositum de la cosa, como amor, odio.

15. Es sabido que no podemos definir hasta el infinito, sino que muchos conceptos deben permanecer indefinidos, muchos pueden ser descompuestos [zergliedern] hasta un cierto grado. Aquí surge la pregunta de si es posible determinar precisamente esos límites, o no. Las razones principales por las cuales hay que medir en el definir son la cantidad de palabras y conceptos de notas simples, la prevención de los círculos al definir, la imposibilidad de definir los conceptos claros, particularmente cuando no son conceptos principales. Imposibilidad de definir esos conceptos que no podemos diferenciar, etc.

Uno define algo cuando muestra su relación con alguna cosa conocida. La relación determina aquello a partir de ésta. Así que si se define para señalar la cosa, deben ser ya conocidas tanto la otra cosa como la relación. Si se define para demostrar, debe indicarse la relación con una cosa [214] de la que se tienen muchos principios. Pero si se define para la praxis, entonces las relaciones deben ser del tipo de las que hemos ejemplificado en $\$ 9$.

Una definición real [Sacherklärung] debe determinar una cosa a partir de tales relaciones con otras que tengamos en nuestro poder y que por lo tanto podamos hacer nosotros mismos. Frecuentemente pueden derivarse de definiciones nominales [Worterklärungen], y en este caso una definición nominal puede descomponerse hasta llegar a las relaciones y partes que están en nuestro poder.

16. Veamos los motivos y las fuentes de las definiciones, en particular, 1. de las definiciones nominales. Para ellas hay, fuera de los medios introducidos arriba (4-9), los siguientes motivos. El uso equivocado de las palabras y su demostración; éste mostrará una determinación esencial del concepto. El origen de la cosa y primer uso de la palabra. La derivación [Herleitung] del significado metafórico. Las locuciones en las que esencialmente figuran las palabras. Es claro en sí mismo que uno tiene que mirar esos motivos, si hay que determinar de la forma más fácil y correcta un concepto dado. 